\title{
Conditional gene expression systems to study herpesvirus biology in vivo
}

\author{
Torsten Sacher $\cdot$ Stefan Jordan $\cdot$ Christian A. Mohr • \\ Aurore Vidy • Annelies M. G. Weyn · Zsolt Ruszics • \\ Ulrich H. Koszinowski
}

Received: 31 January 2008 / Published online: 7 March 2008

(C) Springer-Verlag 2008

\begin{abstract}
Cytomegalovirus (CMV), a prototypic $\beta$-herpesvirus, is an important human pathogen causing protean clinical manifestations in immature and immunocompromised patients. Mechanisms of infection can be studied in a mouse model. Mouse cytomegalovirus (MCMV) resembles in pathogenesis its human counterpart in many ways. Although MCMV infection is studied extensively on the level of organs, the contribution of specific cell types to viral replication in vivo is still elusive. Here we describe our approach based on the the Cre/loxP-system to investigate MCMV infection at the level of cell types in vivo. Using bacterial artificial chromosome (BAC)-technology, we created an MCMV virus containing an enhanced green fluorescent protein (egfp) reporter-gene which is not expressed due to a 'Stop' cassette flanked by two loxP-sites between promoter and coding sequence. Infection of cretransgenic mice with this reporter virus results in the deletion of the 'Stop' cassette and expression of EGFP in a cell type-specific manner. Using this conditional gene expression system we are able to quantify viral productivity in specific cell types and to determine their contribution to viral dissemination in vivo. Furthermore, the deletion of viral genes can be used to screen for cell type-specificity of viral gene functions. Hence, conditional MCMV mutants allow the study of herpesvirus biology on the level of cell types in vivo.
\end{abstract}

T. Sacher and S. Jordan have equally contributed to this work.

T. Sacher · S. Jordan · C. A. Mohr · A. Vidy · A. M. G. Weyn .

Z. Ruszics $\cdot$ U. H. Koszinowski $(\square)$

Max von Pettenkofer-Institute,

Ludwig Maximilians-University,

Pettenkoferstrasse 9a, 80336 Munich, Germany

e-mail: koszinowski@mvp.uni-muenchen.de
Keywords Cytomegalovirus - Conditional mutagenesis . Cre-recombinase $\cdot$ Transgenic mice $\cdot$ Cell type-specificity $\cdot$ Endothelial cells $\cdot$ Hepatocytes

\section{Introduction}

Herpesvirus infections are widespread in the human population. Cytomegalovirus (CMV), a prototypic $\beta$-herpesvirus, is an important human pathogen. Generally, individuals with an immature or compromised immune system, for instance fetuses, premature newborn infants, AIDS patients and transplant recipients frequently develop serious disease $[1,2]$. The clinical manifestations of CMV-disease are diverse, since in absence of immune control virus replication occurs in various tissues.

One common characteristic of the $\beta$-herpesviridae is their pronounced species specificity [1]. Mouse cytomegalovirus (MCMV) has an extensive sequence homology with human CMV (HCMV) [3] and provides a model to study the biology of CMV infection in the living host $[4,5]$. Apart from representing a reliable animal model, MCMV resembles its human counterpart with respect to organ- and cell tropism, pathogenesis during acute infection, establishment of latency, and reactivation after immunosuppression $[4,6,7]$. Thus, MCMV is the model system of choice to get insights into the mechanisms of HCMV pathogenesis. In addition, a wide range of genetically engineered mice offers the opportunity to study the molecular mechanisms of CMV-infection.

Both HCMV and MCMV encompass a sequence of $230 \mathrm{~kb}$, with 165 predicted genes in the case of HCMV [8, 9], while the MCMV genome harbours about 170 ORFs [3]. Although the large genome size complicates the analysis, in recent years new genetic tools simplified mutagene- 
sis in the CMV genome to identify and study gene functions. Particularly the cloning of CMV as infectious bacterial artificial chromosomes (BAC) in E. coli has made the viral genome accessible for forward and reverse genetic approaches [10-12]. Large deletions in the MCMV genome proved 101 ORFs not to be essential for replication [12]. These genes, however, are probably involved in immune modulation and the establishment of infection in different cell types and thus are relevant virulence factors in vivo. For instance, genes M36 and M45 have been found to be important in the infection of macrophages and endothelial cells in vitro [13, 14]. Yet the analysis of cell types in vitro, even if they were available, can only distantly reflect in vivo conditions. Therefore, it is mandatory to investigate in vivo how MCMV genes regulate virus growth in different cells. To target and to investigate cell type-specificity of viral gene products in vivo, new approaches are required. Here we present our approach based on the Cre/loxP-system.

\section{Conditional gene expression to study gene activation and silencing in vivo}

The Cre/loxP-system was the first established recombination system and is currently the most frequently used one $[15,16]$. Cre is the $38 \mathrm{kDa}$ product of the cre (cyclisation recombination) gene of bacteriophage $\mathrm{P} 1$. The Cre enzyme recognizes a $34 \mathrm{bp}$ sequence called loxP (locus of $\mathrm{X}$-over P1) and efficiently catalyzes DNA recombination between pairs of directly repeated loxP sites, resulting in the excision of the DNA in-between as a covalently closed circle $[17,18]$.

About 15 years ago the first $c r e$-transgenic mouse was generated [19]. Since then Cre-mediated recombination of genomic DNA has been proved to be a powerful tool in the study of gene functions in vivo [20]. Both the activation and silencing of gene expression can be regulated in a temporally and spatially controlled manner [15]. To silence genes of interest, an essential region of the gene is flanked by $\operatorname{lox} P$ sites (i.e. a so-called floxed allele). Mating mice that carry a floxed allele with mice expressing Cre under the control of cell type specific promoters will result in progeny in which the floxed allele is deleted in selected tissues (Fig. 1a). These conditional knockout mice made it possible to circumvent early lethal phenotypes. In contrast, tissue-specific gene activation can be achieved if a floxed transcriptional 'Stop' sequence (i.e stop codons and a polyadenylation sequence) is placed between the promoter and the coding sequence keeping the gene dormant. Cell typespecific Cre expression leads to the cleavage of the 'Stop' sequence and activation of gene transcription [20,21]. a

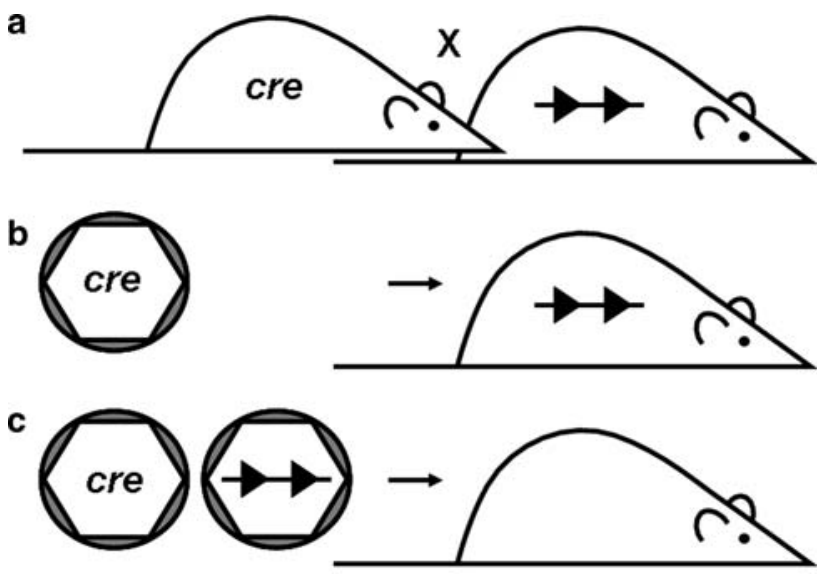

d

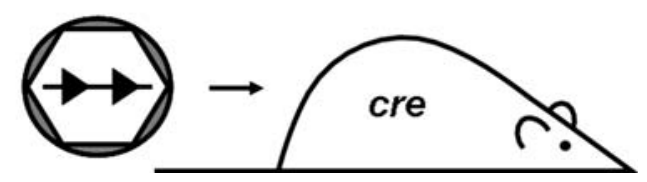

Fig. 1 Building blocks of the Cre/loxP-system in virology. a The 'standard approach' for tissue-specific silencing or activation of mouse genes comprises the mating of cre-transgenic mice with mice harbouring floxed sequences in their genome $[15,16]$. b Using engineered viruses, Cre-expressing viruses mediate recombination of floxed sequences in the genome of infected cells $[22,23]$. $\mathbf{c}$ In co-infection experiments, mice are infected with both a virus containing the cregene and a second virus harbouring floxed sequences [24, 25]. d To regulate expression of virally delivered genes in a cell type-specific manner, cre-transgenic mice are infected with viruses containing floxed sequences $[25,26]$. cre $=$ cre-gene, $\longrightarrow \longrightarrow$ = floxed sequences

\section{Usage of the Cre/loxP-system in virology}

Both the recombination of floxed cellular genes [22, 23] or viral genes $[24,25]$ after infection with a virus that carried the cre gene has been shown. First, an engineered adenovirus delivered Cre in vivo (Fig. 1b) [22]. After infection of mice harbouring floxed sequences in their genome, spleen and liver, the organs with the highest infection load, also showed the highest levels of recombination. Basing on this principle, Moser and colleagues used conditional knockout mice with a floxed DNA methyltransferase 1 (Dnmt 1) gene, an essential factor for the survival of proliferating cells, expressed Cre from a recombinant $\gamma$ MHV68 virus [23] and were able to show that proliferation of B-cells is necessary for the establishment of chronic $\gamma$ MHV68 infection. A Cre expressing adenovirus was also used in coinfection experiments with a second adenovirus mutant containing a floxed 'Stop' cassette followed by the sequence of the murine Fas ligand leading to its ectopic expression in the liver after recombination (Fig. 1c) [24]. More recently, in vivo co-infection of individual cells by different CMV variants could be demonstrated by using a 
Cre expressing virus that activates a reporter gene present in the genome of a second virus mutant [25].

Apart from the opportunity to express Cre from a viral vector, however, it is possible to benefit from the steadily growing range of mouse mutants that express the recombinase in different host tissues. Infecting these mouse lines with conditional virus mutants should result in tissue-specific activation or silencing of virally transduced genes (Fig. 1d). Adenovirus containing a floxed reporter gene provided the proof of concept. Mice expressing Cre under the control of a mammary gland-specific promoter accumulated the reporter in the mammary gland [26]. In an elegant approach, deFalco et al. [27] were able to identify neuronal networks by utilizing mice that express Cre specifically in neurons and a pseudorabies virus that was dependent on Cre-mediated recombination for replication and expression of a reporter. After infection, Cre expressing neurons were labelled. Furthermore, since pseudorabies virus is able to cross multiple synapses, neurons connected to the originally infected cells could be identified. This retrograde tracing of conditional pseudorabies virus from specific neurons offers the opportunity to uncover polysynaptic circuits.

Taken together, the combination of animals and viruses as building blocks of the Cre/lox $P$-system offers a constructive and conceptual approach to answer specific questions on virus and cell biology.

\section{The application of conditional MCMV reporter viruses to cytomegalovirus biology}

MCMV replication occurs in many different tissues in the infected host. It is unclear, which cell types dominate virus production during acute infection and how these cells are controlled by innate and adaptive immunity. To study the role of cell types during viral infections we used the Cre/loxP-system to identify and to quantify viral productivity of selected cell types in vivo (Sacher T, accepted for publication).

Using BAC-technology, we generated a recombinant MCMV reporter virus conditionally expressing the enhanced green fluorescent protein (EGFP) as a reporter under the control of the HCMV major immediate-early promoter (Fig. 2). A floxed 'Stop' cassette between promoter and transgene prevents the expression of the reporter. When the reporter virus infects a cell type that expresses Cre, the viral genome is recombined, the floxed 'Stop' cassette is cleaved and the reporter gene is expressed. Thus, infection of a Cre-expressing cell results in a green fluorescent signal, which can be monitored, whereas after infection of Cre-negative cells no recombination occurs and the reporter stays silent.

Since the recombined locus is stably maintained in the viral progeny, it is possible to track virus transmission to

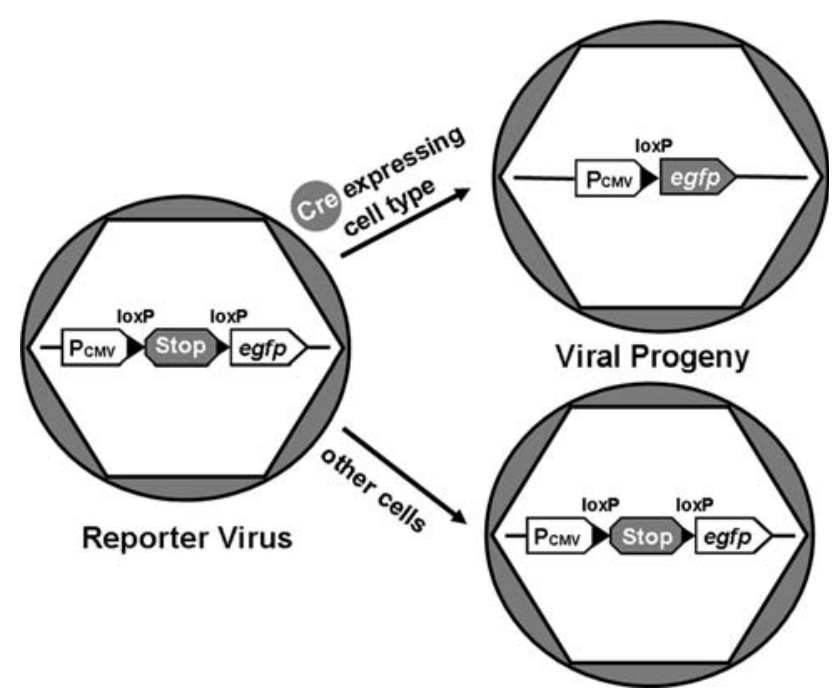

Fig. 2 Cre-mediated recombination of viral genomes. The reporter virus contains a floxed 'Stop' cassette between the egfp coding sequence and the HCMV major immediate early-promoter. After infection of a Cre expressing cell type, the 'Stop' sequence is excised and the recombined reporter locus is maintained in the viral progeny. In other cell types no recombination of the viral genome occurs and produced virus is not genetically labelled

cells that do not express Cre. Dissemination between different cell types within as well as between organs and the colonization of distant targets can thus be studied.

The amount of infectious progeny produced by a specific cell type can be quantified in a standard plaque assay (Fig. 3). While the total number of plaques allows the calculation of virus load of the organ, the amount of green fluorescent plaques gives an estimate of the contribution of the Cre expressing cells to the overall viral productivity. Depending on the aim of the study, it is important to choose the proper time point post infection before assaying the organs. Only after the first viral replication cycle the number of EGFP positive plaques truly reveals the productivity of the Cre expressing cell type. At later time points, virus with a recombined reporter locus allows the tracking of viral progeny between different cell types and dissemination between organs.

The conceptual basis of the experimental approach is simple. However, several points have to be taken into account for a meaningful application and interpretation of conditional reporter viruses.

\section{Marker virus properties}

In principle, the experimental approach is suitable to study not only the biology of MCMV, but of any DNA-virus equipped with a reporter sequence that infects mice and replicates in the nucleus (since in most cre-transgenic mouse strains the recombinase is targeted to the cell 


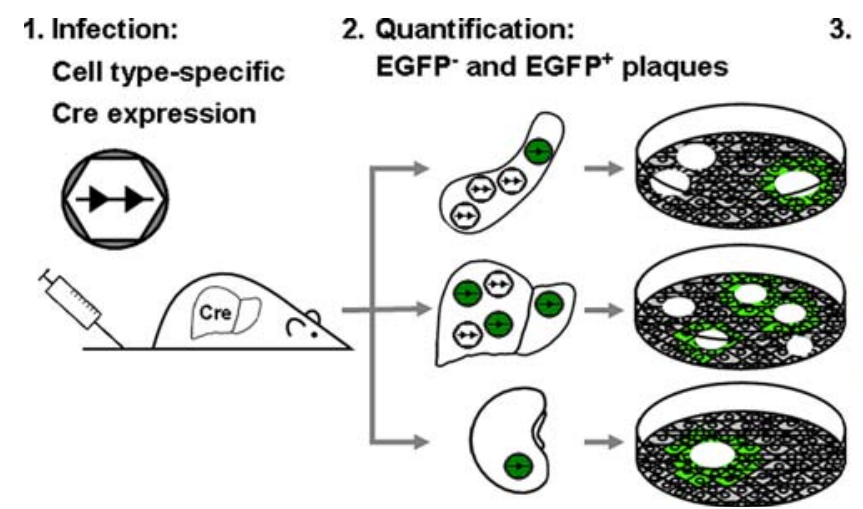

3. Results:

Cell type-specific productivity

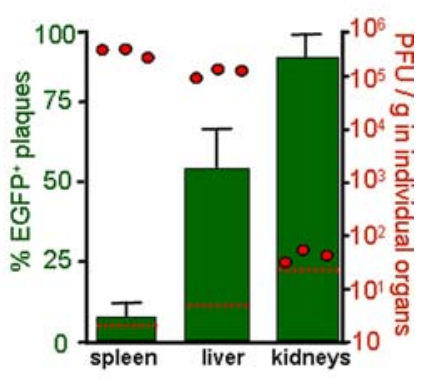

Fig. 3 Experimental setup to address viral productivity and dissemination of specific cell types in vivo. 1. Transgenic mice expressing the Cre recombinase under the control of a cell type-specific promoter are infected with the conditional reporter virus. 2 . After collecting organs of interest, the amount of $\mathrm{EGFP}^{+}$and $\mathrm{EGFP}^{-}$plaque forming units

nucleus), as for instance adenoviruses and herpesviruses (e.g. MHV68 and pseudorabies virus). Clearly, DNAviruses that replicate in the cytoplasm, for example mouse pox virus (ectromelia virus), would require transgenic mice with the Cre enzyme present in the cytosol.

Additional requirements have to be fulfilled for a meaningful application of the Cre/lox $P$-system in virology. First, it is essential that the in vivo replication properties of the conditional reporter virus are independent of whether or not the reporter gene locus is recombined. Second, the expression of Cre in the transgenic mouse strain used should be restricted to the cell type under investigation. And third, a high — or at least a stable ratio — of the genomes produced by a Cre expressing cell should be recombined.

The viral genome cannot exceed a certain length. Therefore, the reporter construct has to replace a viral sequence resulting in a deletion mutant. We chose to insert the reporter into gene $m 157$ yielding the virus MCMV- $\Delta m 157$ flox-egfp. The gene product of $m 157$ is known to be a ligand for the activating NK cell receptor Ly49H [28-30]. Mouse strains expressing Ly49H (e.g. C57BL/6) show a higher resistance to MCMV infection than Ly49H negative mice (e.g. BALB/c). The targeted deletion of $m 157$ avoids the innate immune control mediated by $\mathrm{Ly} 49 \mathrm{H}$ positive NK cells in C57BL/6 mice. The gene function of $m 157$ is also lost in vivo after passage through $\mathrm{Ly} 49 \mathrm{H}$ positive mice and is absent in MCMV strains isolated from wildtype mice. Furthermore, Ly49H is uncommon in laboratory and wildtype mice [31].

Mutant viruses harbouring foreign genes run the risk of not reflecting the physiological reality. Already the mere length of a reporter gene introduced into the viral genome may impair virus fitness and result in attenuation. Also, it is conceivable that the expression of the marker might result in lower titers of the virus with a recombined locus than
(PFU) can be quantified in a standard plaque assay on permissive cells using fluorescent microscopy. 3. Total virus load per organ and mean percentage of $\mathrm{EGFP}^{+}$plaques per experimental group are displayed as red dots and green columns, respectively. Red dashed line detection limit

with an unrecombined genome, for instance due to immune recognition of the marker. In this case, the virus titer of the recombined derivative MCMV- $\Delta m 157$-rec-egfp would not reflect the proportion of virus produced by a certain cell type in comparison to the parental virus MCMV- $\Delta m 157$ flox-egfp. We infected immunocompetent C57BL/6 mice with MCMV- $\Delta m 157-f l o x-e g f p$ and MCMV- $\Delta m 157-$ recegfp and found that both viruses replicated within the first 10 days to comparable titers in different organs. It is not excluded, however, that there exist minor differences as indicated by slightly differing lung titers. Yet, such differences may become important variables in studies over a period of many days. As expected, in the absence of Cre no activation of the reporter gene could be detected. Once Cremediated recombination had occurred, the reporter gene was expressed absolutely reliably, as was shown by $100 \%$ EGFP positive plaques in C57BL/6 mice infected with MCMV- $\Delta m 157-$ rec-egfp. Thus, the conditional MCMV reporter viruses proved to be suitable tools to study virus production and dissemination in vivo.

\section{Different conditions for Cre-mediated host and viral genome recombination}

Originally, cre-transgenic mice were developed to control conditional gene expression in murine tissues. For in vivo gene silencing only one or two floxed alleles per cell have to be cleaved and there is ample time during organogenesis to initiate and accomplish the recombination event. During virus replication, however, the presence of the parental viral genome in the nucleus is a matter of about $12 \mathrm{~h}$; therefore recombination has to occur early and fast. In this situation the Cre enzyme has to be present in sufficient amounts in the nucleus prior to infection. As we find marker expression 
already 8 hours after infection, we presume that the recombination of the reporter virus genome takes place before virus DNA replication starts. In addition, only few infected cells are found in most organs by histological examinations. Therefore, we think that each primary target cell is infected probably by only one virus. The recombination occurs only on one single virus genome per infected cell.

There are possibilities to control that the results obtained are independent of Cre expression levels. First, for some cell types different Cre expressing mouse strains are available. For instance, in both Tie2-cre and Tek-cre mice the same promoter/enhancer combination was used to drive Cre expression specifically in vascular endothelial cells [32, 33]. Since these strains should only differ in the copy number and the insertion site of the cre gene, comparative analysis of these independent strains might provide information whether different Cre expression levels have an influence on the experimental outcome. Second, infection of mice either hemi- or homozygous for the cre transgene with the reporter viruses reveals whether Cre expression operates at or beyond the critical level for efficient recombination. Third, for some cell types more than one specific promoter has been used to generate cre-transgenic mice. For hepatocyte-specific Cre expression, for example, Alb-cre as well as Alfp-cre mice are available that express Cre under the control of the albumin promoter and albumin regulatory elements plus $\alpha$-fetoprotein enhancers, respectively [34, 35]. Comparison of both mouse strains might be helpful.

In general, probably the best evidence that the Cre expression level is sufficient to mediate efficient recombination in a certain cell type is provided when at early time points following infection close to $100 \%$ of the progeny is recombined. This result, however, is only possible if the cell type under study is also the major producer cell type in a given tissue. Yet low proportions of recombined virus may not be due to unsuccessful recombination but may rather reflect unproductive cell types. Our approach presented so far was aimed at a positive identification of cell types important for virus production and dissemination.

\section{Selectivity and stability of Cre expression in cell types and organs}

In order to identify a certain cell type as a virus producer by the Cre/lox $P$-system, the Cre expression has to be restricted to this cell type. Unfortunately, the cell type-specific expression of Cre in transgenic mouse lines is usually examined in the absence of infection and inflammation. A problem may arise if the promoter driving Cre expression is also active in other cell types during inflammation. In this case, an unexpected cell type would contribute to the recombined reporter virus fraction. Therefore, Cre expres- sion in third party cells should not occur in the presence of infection and inflammation. To date this possibility cannot be entirely excluded. Next, different transgenic mouse lines can vary in the reliability of cell type-specific Cre expression. A just transient Cre expression in multiple cell types during embryogenesis, as for instance observed in Tie2-cre mice [32], does not represent an objection to our approach. In our case not the developmental Cre expression but only the actual cell type-specific Cre expression in endothelial cells during infection with the reporter virus is crucial for the experiment. Altogether, the conditions of Cre expression for host gene activation or silencing need not reflect the situation required for viral marker expression.

In the example reported by deFalco and colleagues mentioned above [27] the recombination of any number of virus genomes sufficed to label the infected cell. In contrast to this qualitative approach we aim to use the Cre/loxP-system for a quantitative approach. We count the number of viral progeny with a recombined genome and estimate the viral productivity of a defined cell type. Which factors could account for differences in the proportion of recombined reporter virus found in an organ? Differences in the proportion of recombined reporter virus found between different Cre positive cell types constitute the basic read-out of our experimental system. They give an estimate of the contribution of a given cell type to the overall viral load of an organ, provided that the analysis is restricted to a single viral replication cycle. First of all, the level of Cre should suffice to recombine all genomes, which contribute to production of progeny. Besides this, the size of the virus progeny with a recombined genome depends on whether the cell type under investigation is highly or poorly productive. Differences in the proportion of recombined virus between organs do not necessarily reflect physiologic differences in cell type-specific viral productivity, but rather the different numbers of the Cre expressing cells in different tissues. After infection of Tie2-cre mice, for instance, the viral genome is replicated and recombined in endothelial cells. Some organs possess more endothelial cells than others, which may result in a higher proportion of virus with a recombined genome. Virus in the blood must cross the endothelial cell barrier to infect parenchymal tissues. If the endothelium is fenestrated or discontinuous, however, the reporter virus might cross the endothelial barrier and enter the parenchyma of the organ even without experiencing recombination. Indeed, in Tie2-cre mice organs with fenestrated or discontinuous endothelium like kidney, adrenal glands and liver generally exhibit lower proportions of recombined virus. The same would be true if the virus just passes the endothelial barrier by transcytosis without replication in endothelial cells.

Recombination of the reporter locus in the cell type under investigation should occur with high efficiency, prior 
to DNA replication. There are factors that should have an effect on the level of Cre expression. First of all, the copy number of the cre-transgene harboured by a mouse genome is important. Whereas mice generated by knock-in technology contain one to two cre copies, transgenic mouse strains may carry multiple copies of the cre gene. In direct comparison, the Cre expression levels of the former should be lower. In initial experiments using conditional reporter viruses, we studied CD11c-cre mice and CD45-cre mice. CD45 is a pan-leukocyte marker and CD11c is predominantly expressed on dendritic cells. Therefore, if not even higher in CD45-cre mice, the recombination levels in both mouse strains should at least be equal. To our surprise, however, we could not find progeny with a recombined reporter locus in CD45-cre mice, while in CD11c-cre mice a considerable amount of recombined reporter virus was found. The fact that the cre gene was introduced into the genome of CD45-cre mice by knockin technology, whereas the CD11c-cre mouse is a transgenic strain, might explain these apparently contradictory results. We feel that the level of Cre expression in knock-in mice is usually not high enough to mediate efficient recombination of the virus genome. Unfortunately, some mice engineered to express Cre in a cell type-specific manner are only available as knock-in strains.

Even if in a transgenic mouse strain Cre cell type-specific expression is proven, as for instance in endothelial cells in Tie2-cre mice, the constitutive Cre expression levels may vary between endothelial cells in different organs. For example, endothelial cells that are found in the sinusoids of the liver are morphologically and phenotypically distinct from endothelial cells that line the large vessels (e.g. the aorta) [36, 37] and this may extend to constitutional promoter activities.

As discussed above, virus induced inflammation can activate Cre expression in unexpected cell types. Similarly, the infection may modulate the activity of promoters which drive Cre expression. This would lead to altered levels of Cre recombinase during infection and inflammation. For instance, usually the albumin promoter is used for cell typespecific Cre expression in hepatocytes [34]. Yet, it was shown that MCMV infected hepatocytes produce less albumin [38], and Cre expression levels during infection may in fact be reduced.

\section{Identification of cell type-specific functions of viral gene products}

Although the application of MCMV reporter viruses in the Cre/loxP-system is a powerful tool to identify virus productive cells, there are still apparent difficulties in comparing different transgenic mouse lines with regard to constitutive and inflammation induced Cre expression and with regard to the number of Cre expressing cells in a particular organ. The prospects are much better when a comparison of different virus mutants in one and the same cretransgenic strain is the objective. Genes M36 and M45 were already found to be important in the infection of macrophages and endothelial cells in vitro [13, 14]. It is conceivable that a proportion of MCMV's 101 ORFs which are not essential for replication play a role in governing virus-cell interactions in the living host $[12,39]$. On the basis of the BAC-cloned MCMV- $\Delta m 157$-flox-egfp genome, mutants can easily be generated with deletions in additional genes resulting in viruses MCMV- $\Delta m 157$-flox-

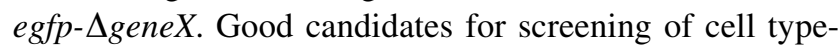
specific functions for example would be individual members of the m145 gene family and gene $M 27$, genes which are involved in NK cell regulation and interferon response modulation, respectively [40-42]. Furthermore, it would be of interest to test published mutants that do not seem to have any phenotype in vivo. For example, viruses mutated in genes $m 09, M 25$ and $m 128$ (ie2) exhibited a titer similar to that of the wildtype virus in the salivary glands, lungs, liver, spleen and kidneys of BALB/c mice [43-45]. M43 mutants also did not show any replication difference in most organs, except in the salivary gland [46]. The same total titer, however, could result from the contribution of different producer cells. The inability to produce progeny in one cell type might be compensated by the productivity in another cell type. A cell type might be poor as a virus producer but highly active as a stimulator of the immune response. If this cell type is excluded from productive infection, the indirect effects of the reduced inflammation might surpass the loss of direct productivity.

After infection of cre-transgenic mice with a virus mutant MCMV- $\Delta m 157$-flox-egfp- $\Delta$ geneX and the parental virus MCMV- $\Delta m 157$-flox-egfp, the shift in the proportion of virus with a recombined locus would indicate a function of gene $X$.

Tie2-cre mice expressing the Cre recombinase in endothelial cells were infected with MCMV- $\Delta m 157$-flox-egfp$\Delta g e n e A$ and MCMV- $\Delta m 157$-flox-egfp (Fig. 4). The example shows that a deletion of gene $A$ results in a reduced virus titer in the spleen. The proportion of gene $A$-deficient virus derived from endothelial cells, however, accounted for more than $90 \%$ of the spleen's total virus load compared to only $50 \%$ in the case of virus with an intact gene $A$. Thus, in the case of MCMV- $\Delta m 157$-flox-egfp- $\Delta$ geneA almost every virus particle found in the spleen now originated from endothelial cells. This demonstrates a clear cell typespecific effect for gene $A$. We suppose that gene $A$ is required for virus replication in the major producer cells of the spleen, which still need to be identified. Apparently, gene $A$ is not essential for replication in endothelial cells 

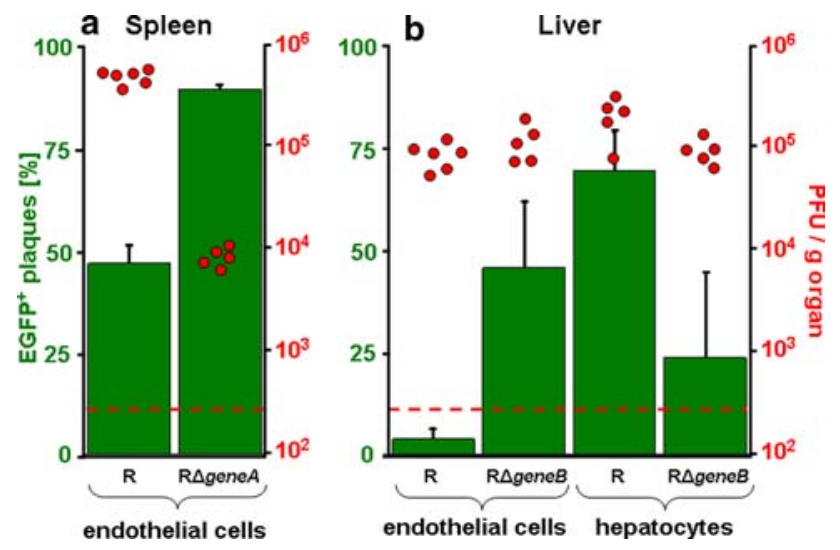

Fig. 4 Cell type-specific functions of viral gene products in vivo. Cell type-specific effects were found when the parental MCMV reporter virus $\Delta m 157$-flox-egfp $(\mathrm{R})$ was compared with the deletion mutants $\mathrm{R} \Delta$ gene $A$ and $\mathrm{R} \Delta$ gene $B$ in $\mathbf{a}$ the spleen and $\mathbf{b}$ the liver, respectively. Organs were assayed at day 3 p.i. a Tie2-cre mice expressing Cre specifically in endothelial cells were infected with $10^{6} \mathrm{PFU}$ of R and R $\Delta$ geneA. The green columns represent the proportion of virus originating from endothelial cells. b Tie2-cre mice and Alb-cre mice were infected with $10^{6} \mathrm{PFU}$ of $\mathrm{R}$ and $\mathrm{R} \Delta$ gene $B$. The green columns represent the proportion of virus produced by endothelial cells and hepatocytes, respectively. Red dots total virus titer, red dashed line detection limit

and this explains the high proportion of recombined gene $A$-deficient virus.

A similar experiment was performed in Tie2-cre mice with the deletion mutant MCMV- $\Delta m 157$-flox-egfp- $\Delta g e$ $n e B$. Here, the total virus titer in the liver remained stable at day 3 post infection, but the fraction of gene $B$-deficient virus progeny increased more than tenfold indicating that the quantity of virus produced by endothelial cells was significantly augmented. In the complementary experiment, Alb-cre mice were infected with MCMV- $\Delta m 157$-flox-egfp$\Delta g e n e B$ and MCMV- $\Delta m 157$-flox-egfp. As expected, no changes in the total liver virus titer could be detected. However, the amount of virus produced by hepatocytes decreased. Thus, by deletion of gene $B$, virus productivity in the liver shifted from hepatocytes to endothelial cells. Clearly, such a phenotype cannot be detected by the use of conventional deletion mutants that do not allow cell typespecific tracing of viral progeny.

\section{Future applications of conditional gene expression to study herpesvirus biology in vivo}

Conditional MCMV reporter viruses can be used to trace virus production and dissemination as well as to identify cell type-specific functions of viral gene products in vivo. We hope that the spectrum of applications can be further expanded. Depending on the question, the conditionally expressed marker can be customized. For instance, it is possible to express the firefly luciferase after Cre-mediated recombination instead of EGFP. Luciferase-dependant bioluminescence can be detected by noninvasive methods and the dynamics of infection can therefore be studied on the level of organs as a single mouse kinetics.

Furthermore, conditional gene expression by MCMV mutants is not just restricted to reporter genes. For instance, MCMV viruses can be engineered to express model antigens after Cre-mediated recombination. Upon infection of transgenic mice with MCMV- $\Delta m 157$-flox-ovalbumin, antigenic markers will be expressed in Cre positive cell types and presented on MHC complexes. If it is possible to restrict antigen expression to the Cre positive cells, questions of direct and cross presentation by different cell types become amenable.

In summary, viral productivity in vivo was so far only accessible on the level of organs. The application of conditional reporter viruses in the Cre/lox $P$-system makes it now possible to study viral productivity, virus dissemination, antigen presentation, and immune modulation at the level of cell types in vivo.

Acknowledgments S.J. and A.V. were supported by a research fellowship of the Studienstiftung des deutschen Volkes and the Humboldt Stiftung, respectively. U.H.K. and Z.R. were supported by the DFG (SFB 455 A7) and U.H.K by NGFN grant 01 GS0405 and the Bayerische Forschungsstiftung. We thank J. Gregor and N. Röder for expert technical assistance and are indebted to S. Schultz for her support on graphic design.

\section{References}

1. Britt W (2006) Human cytomegalovirus infections and mechanisms of disease. In: Reddehase MJ (ed) Cytomegaloviruses. Molecular biology and immunology. Caister Academic Press, Norfolk, pp 1-28

2. Mocarski ES, Shenk T, Pass RF (2007) Cytomegaloviruses. In: Knipe DM, Howley PM (eds) Fields virology. Lippincott Williams and Wilkins, Philadelphia, pp 2701-2773

3. Rawlinson WD, Farrell HE, Barrell BG (1996) Analysis of the complete DNA sequence of murine cytomegalovirus. J Virol 12:8833-8849

4. Mocarski ES Jr, Kemble GW (1996) Recombinant cytomegaloviruses for study of replication and pathogenesis. Intervirology 56:320-330

5. Kern ER (1999) Animal models for cytomegalovirs infection: Murine CMV. In: Zak O, Sande M (eds) Handbook of animal models of infection. Academic Press, London, pp 927-934

6. Reddehase MJ, Podlech J, Grzimek NK (2002) Mouse models of cytomegalovirus latency: overview. J Clin Virol 25(Suppl 2):S23S36

7. Krmpotic A, Bubic I, Polic B, Lucin P, Jonjic S (2003) Pathogenesis of murine cytomegalovirus infection. Microbes Infect 13:1263-1277

8. Murphy E, Yu D, Grimwood J, Schmutz J, Dickson M, Jarvis MA, Hahn G, Nelson JA, Myers RM, Shenk TE (2003) Coding potential of laboratory and clinical strains of human cytomegalovirus. Proc Natl Acad Sci USA 25:14976-14981

9. Dolan A, Cunningham C, Hector RD, Hassan-Walker AF, Lee L, Addison C, Dargan DJ, McGeoch DJ, Gatherer D, Emery VC, 
Griffiths PD, Sinzger C, McSharry BP, Wilkinson GWG, Davison AJ (2004) Genetic content of wild-type human cytomegalovirus. J Gen Virol 5:1301-1312

10. Messerle M, Crnkovic I, Hammerschmidt W, Ziegler H, Koszinowski UH (1997) Cloning and mutagenesis of a herpesvirus genome as an infectious bacterial artificial chromosome. Proc Natl Acad Sci USA 26:14759-14763

11. Wagner M, Koszinowski UH (2004) Mutagenesis of viral BACs with linear PCR fragments (ET recombination). Methods Mol Biol 256:257-268

12. Brune W, Wagner M, Messerle M (2006) Manipulating cytomegalovirus genomes by bac mutagenesis: strategies and applications. In: Reddehase MJ (ed) Cytomegaloviruses. Molecular biology and immunology. Caister Academic Press, Norfolk, pp 64-89

13. Brune W, Menard C, Heesemann J, Koszinowski UH (2001) A ribonucleotide reductase homolog of cytomegalovirus and endothelial cell tropism. Science 5502:303-305

14. Menard C, Wagner M, Ruzsics Z, Holak K, Brune W, Campbell AE, Koszinowski UH (2003) Role of murine Cytomegalovirus US22 gene family members in replication in macrophages. J Virol 10:5557-5570

15. Lewandoski M (2001) Conditional control of gene expression in the mouse. Nat Rev Genet 10:743-755

16. Feil R, Metzger D (2007) Conditional mutagenesis: an approach to disease models. Springer, Berlin

17. Sternberg N, Hamilton D (1981) Bacteriophage P1 site-specific recombination : I. Recombination between loxP sites. J Mol Biol 4:467-486

18. Sauer B, Henderson N (1988) Site-Specific DNA Recombination in Mammalian Cells by the Cre Recombinase of Bacteriophage P1. Proc Natl Acad Sci 14:5166-5170

19. Lakso M, Sauer B, Mosinger B Jr, Lee EJ, Manning RW, Yu SH, Mulder KL, Westphal H (1992) Targeted oncogene activation by site-specific recombination in transgenic mice. Proc Natl Acad Sci USA 14:6232-6236

20. Sauer B (1998) Inducible gene targeting in mice using the Cre/loxSystem. Methods 4:381-392

21. Orban PC, Chui D, Marth JD (1992) Tissue- and site-specific dna recombination in transgenic mice. Proc Natl Acad Sci 15:68616865

22. Wang Y, Krushel LA, Edelman GM (1996) Targeted DNA recombination in vivo using an adenovirus carrying the cre recombinase gene. Proc Natl Acad Sci 9:3932-3936

23. Moser JM, Upton JW, Allen RD III, Wilson CB, Speck SH (2005) Role of B-Cell proliferation in the establishment of gammaherpesvirus latency. J Virol 15:9480-9491

24. Zhang HG, Bilbao G, Zhou T, Contreras JL, Gomez-Navarro J, Feng M, Saito I, Mountz JD, Curiel DT (1998) Application of a fas ligand encoding a recombinant adenovirus vector for prolongation of transgene expression. J Virol 3:2483-2490

25. Cicin-Sain L, Podlech J, Messerle M, Reddehase MJ, Koszinowski UH (2005) Frequent coinfection of cells explains functional in vivo complementation between cytomegalovirus variants in the multiply infected host. J Virol 15:9492-9502

26. Wagner KU, Wall RJ, St Onge L, Gruss P, Wynshaw-Boris A, Garrett L, Li M, Furth PA, Hennighausen L (1997) Cre-mediated gene deletion in the mammary gland. Nucleic Acids Res 21:4323-4330

27. DeFalco J, Tomishima M, Liu H, Zhao C, Cai X, Marth JD, Enquist L, Friedman JM (2001) Virus-assisted mapping of neural inputs to a feeding center in the hypothalamus. Science 5513:2608-2613

28. Arase H, Mocarski ES, Campbell AE, Hill AB, Lanier LL (2002) Direct recognition of cytomegalovirus by activating and inhibitory NK cell receptors. Science 5571:1323-1326

29. Smith HRC, Heusel JW, Mehta IK, Kim S, Dorner BG, Naidenko OV, lizuka K, Furukawa H, Beckman DL, Pingel JT, Scalzo AA, Fremont DH, Yokoyama WM (2002) Recognition of a virus-en- coded ligand by a natural killer cell activation receptor. Proc Natl Acad Sci 13:8826-8831

30. Bubic I, Wagner M, Krmpotic A, Saulig T, Kim S, Yokoyama WM, Jonjic S, Koszinowski UH (2004) Gain of virulence caused by loss of a gene in murine cytomegalovirus. J Virol 14:7536-7544

31. Voigt V, Forbes CA, Tonkin JN, Degli-Esposti MA, Smith HR, Yokoyama WM, Scalzo AA (2003) Murine cytomegalovirus m157 mutation and variation leads to immune evasion of natural killer cells. Proc Natl Acad Sci USA 23:13483-13488

32. Constien R, Forde A, Liliensiek B, Grone HJ, Nawroth P, Hammerling G, Arnold B (2001) Characterization of a novel EGFP reporter mouse to monitor Cre recombination as demonstrated by a Tie2 Cre mouse line. Genesis 1:36-44

33. Koni PA, Joshi SK, Temann UA, Olson D, Burkly L, Flavell RA (2001) Conditional vascular cell adhesion molecule 1 deletion in mice: impaired lymphocyte migration to bone marrow. J Exp Med 6:741-754

34. Postic C, Shiota M, Niswender KD, Jetton TL, Chen Y, Moates JM, Shelton KD, Lindner J, Cherrington AD, Magnuson MA (1999) Dual roles for glucokinase in glucose homeostasis as determined by liver and pancreatic beta-cell-specific gene knock-outs using Cre recombinase. J Biol Chem 1:305-315

35. Kellendonk C, Opherk C, Anlag K, Schutz G, Tronche F (2000) Hepatocyte-specific expression of Cre recombinase. Genesis 2:151-153

36. Limmer A, Ohl J, Kurts C, Ljunggren HG, Reiss Y, Groettrup M, Momburg F, Arnold B, Knolle PA (2000) Efficient presentation of exogenous antigen by liver endothelial cells to CD8+ T cells results in antigen-specific T-cell tolerance. Nat Med 12:1348-1354

37. Knolle PA, Limmer A (2001) Neighborhood politics: the immunoregulatory function of organ-resident liver endothelial cells. Trends Immunol 8:432-437

38. Fang F, Nie X, Li G (1999) A model system of primary murine hepatocytes infected by murine cytomegalovirus. J Tongji Med Univ 3:185-189

39. Cicin-Sain L, Bubic I, Schnee M, Ruzsics Z, Mohr C, Jonjic S, Koszinowski UH (2007) Targeted deletion of regions rich in immune-evasive genes from the cytomegalovirus genome as a novel vaccine strategy. J Virol 24:13825-13834

40. Hasan M, Krmpotic A, Ruzsics Z, Bubic I, Lenac T, Halenius A, Loewendorf A, Messerle M, Hengel H, Jonjic S, Koszinowski UH (2005) Selective down-regulation of the NKG2D ligand H60 by mouse cytomegalovirus m155 glycoprotein. J Virol 5:2920-2930

41. Krmpotic A, Hasan M, Loewendorf A, Saulig T, Halenius A, Lenac T, Polic B, Bubic I, Kriegeskorte A, Pernjak-Pugel E, Messerle M, Hengel H, Busch DH, Koszinowski UH, Jonjic S (2005) NK cell activation through the NKG2D ligand MULT-1 is selectively prevented by the glycoprotein encoded by mouse cytomegalovirus gene m145. J Exp Med 2:211-220

42. Zimmermann A, Trilling M, Wagner M, Wilborn M, Bubic I, Jonjic S, Koszinowski U, Hengel H (2005) A cytomegaloviral protein reveals a dual role for STAT2 in IFN- $\gamma$ signaling and antiviral responses. J Exp Med 10:1543-1553

43. Zhan X, Lee M, Xiao J, Liu F (2000) Construction and characterization of murine cytomegaloviruses that contain transposon insertions at open reading frames m09 and M83. J Virol 16:7411-7421

44. Zhan X, Lee M, Abenes G, Von RI, Kittinunvorakoon C, RossMacdonald P, Snyder M, Liu F (2000) Mutagenesis of murine cytomegalovirus using a Tn3-based transposon. Virology 2:264-274

45. Cardin RD, Abenes GB, Stoddart CA, Mocarski ES (1995) murine cytomegalovirus IE2, an activator of gene expression, is dispensable for growth and latency in mice. Virology 1:236-241

46. Xiao J, Tong T, Zhan X, Haghjoo E, Liu F (2000) In vitro and in vivo characterization of a murine cytomegalovirus with a transposon insertional mutation at open reading frame M43. J Virol 20:9488-9497 